Published online 2017 April 13.

Abstract

\title{
Sonographic and Imaging of Grunulomatous Disease of Breast
}

\author{
Ahmad Soltani Shirazi ${ }^{1, *}$ \\ ${ }^{1}$ Jundishapour Univesity of Medical Sciences \\ "Corresponding author: Ahmad Soltani Shirazi, Jundishapour Univesity of Medical Sciences. E-mail: dr.a.soltanishirazi@gmail.com
}

Received 2016 December 21; Accepted 2017 February 08.

\begin{abstract}
Idiopathic lobular grunulomatous mastitis is a chronic inflammatory disease of Breast that can clinically mimic Brent carcinoma or pyogenic abscess. The most common clinical presentation is a fine unilateral discrete mass associated Inflammation of overlying skin. Nipple retraction and sinus formation are present. Breast TB should be considered in differential we present a few cause of our center with sonography findings and lab test data. The most common mammographic findings an asymmetric density or ill-defined mass. Ultrasound findings are variable irregular hypoecho mass and cyst with thick irregular wall. The cyst wall and septation show imageble flow on color Doppler images. Fistule formation with echogenic fluid and imageble flow of fistule ducts are seen in $60 \%$ of patient. In our cases $15 \%$ have ductal ectesia with abscess $85 \%$ thick irregular wall multiple complex cysts and $65 \%$ echogenic fluid collection and fistula tract.
\end{abstract}

This is an abstract presented in the 33rd Iranian congress of radiology (ICR) and the 15th congress of Iranian radiographic science association (IRSA). 